\title{
Vers l'éradication mondiale de la poliomyélite
}

\section{Michel Rey}

M. Rey: Professeur émérite de Maladies infectieuses et tropicales à la Faculté de Médecine de Clermont-Ferrand, Président de la Commission nationale de certification de l'éradication de la poliomyélite. 5, boulevard du Montparnasse, 75006 Paris, France.

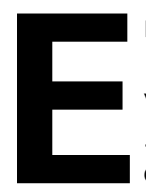

ncouragée par le succès de l'éradication mondiale de la variole, certifiée en 1980, 3 ans après le dernier cas, observé en Somalie, I'Organisation Mondiale de la Santé (OMS) a lancé en 1988 le programme d'éradication mondiale de la poliomyélite. Ce programme est le prolongement du programme élargi de vaccinations, lancé en 1974, dont la vaccination généralisée des jeunes enfants contre la poliomyélite est I'un des éléments. A cette date il était estimé que la polio provoquait chaque année dans le monde environ 500000 nouveaux cas annuels d'infirmités motrices définitives [1]. Fléau bientôt historique de I'humanité, la polio, maladie peu meurtrière, est essentiellement une maladie invalidante. Ce fut la cause majeure des infirmités motrices définitives, autrefois dans tous les pays y compris les pays industrialisés, encore récemment dans la plupart des pays en développement. Quand a été lancé le programme d'éradication, la prévalence mondiale des invalidités imputables à la poliomyélite était de l'ordre de 20 millions [2].

L'éradication mondiale de la poliomyélite a été estimée à juste titre comme techniquement et opérationnellement réalisable, du fait de ses caractéristiques épidémiologiques, et de la disponibilité d'une vaccination efficace. Les trois poliovirus sont exclusivement humains, et leur survie est relativement brève, chez les porteurs asymptomatiques (de loin les plus fréquents) ou malades, ainsi que dans l'environnement. L'éradication de la polio se justifie non seulement sur le plan humain, mais aussi d'un point de vue économique: son obtention ferait économiser chaque année 1,5 milliard de dollars US [2].

\section{Le programme de I'OMS et les résultats obtenus à ce jour}

Ayant mobilisé tous les pays du monde, le programme d'éradication, défini et piloté par l'OMS, a été très largement soutenu par I'UNICEF, et a bénéficié d'aides substantielles de plusieurs pays, ainsi que de la Banque mondiale, du CDC, de I'USAID, du Rotary international et de quelques autres donateurs.

La stratégie recommandée a d'abord été basée sur le renforcement de la vaccination généralisée.par le vaccin oral, ajoutant à la vaccination de routine des jeunes enfants des campagnes de masse périodiques. En effet la vaccination de routine réalisée dans le cadre du PEV ne touche en moyenne, depuis 1990, que $80 \%$ des jeunes enfants dans les pays en développement, et s'est avérée incapable, avec ce taux insuffisant de couverture vaccinale, de faire disparaître la circulation des poliovirus.

C'est le vaccin vivant atténué de Sabin, administré oralement, qui a été recommandé par l'OMS. Peu onéreux, facile à administrer, ce vaccin a l'avantage $d$ 'induire une immunité locale, s'opposant à la pénétration digestive des virus. Par ailleurs, les vaccinés sont contagieux, et peuvent communiquer le virus vaccin à leur entourage, élargissant ainsi la couverture vaccinale. Pour l'ensemble des pays, qui ont appliqué le vaccin oral, les avantages de celui-ci l'emportent largement sur ses inconvénients, tels que sa thermosensibilité, ses effets indésirables (survenue, à vrai dire très rare, d'accidents neurologiques postvaccinaux, attribués à une réversion du virus vaccin vers la virulence) auxquels s'ajoute une réduction de son efficacité, constatée dans les pays tropicaux (la séroconversion obtenue vis-à-vis du poliovirus 1 par 3 doses successives serait de l'ordre de $75 \%$ $85 \%)$.

De son côté, le vaccin inactivé injectable de Salk s'est montré capable d'éliminer la polio dans les pays industrialisés qui l'ont utilisé exclusivement 
(Pays-bas, Suède, Finlande) ou presque exclusivement (France). Très efficace et très bien toléré, il est progressivement introduit dans les programmes de vaccination des pays industrialisés.

Le renforcement de la stratégie vaccinale a consisté à ajouter à la vaccination de routine des campagnes de masse sous la forme de Journées nationales de vaccination (sous la forme de deux campagnes annuelles, à un mois d'intervalle), expérimentées avec succès à Cuba puis au Brésil, mises en place actuellement dans la plupart des pays encore endémiques. Le deuxième volet de cette stratégie est le renforcement de la surveillance clinique, virologique, épidémiologique de l'infection poliomyélitique. La surveillance de tous les cas de paralysie flasque aiguë observés chez les moins de 15 ans (détection, notification et investigation virologique, sur un double prélèvement de selles) est la stratégie recommandée par I'OMS. La plupart des pays, industrialisés ou en développement, se sont efforcés de l'appliquer, non sans difficultés. Plusieurs pays industrialisés, dont la France, ont préféré renforcer la surveillance virologique des entérovirus, d'une part chez les malades de tout âge présentant des symptomes évocateurs (neurologiques ou autres), et d'autre part, si possible, dans l'environnement (eaux usées).

A la suite de I'application de ce programme, le recul de la polio a été spectaculaire dans la plupart des pays. En 1991 a été observé au Pérou le dernier cas détecté et confirmé sur le continent américain, qui a été certifié exempt de poliomyélite en 1994. Le dernier cas observé dans la région du Pacifique occidental (au Cambodge) date de 1997, le dernier cas observé (en Turquie) dans la région européenne (qui comprend l'exURSS) date de 1998. La polio est considérée comme éliminée de la majorité du continent asiatique, à I'exception notable des pays du souscontinent indien, et de I'Irak. En Afrique, le Maghreb, I'Afrique australe, I'Afrique de I'Est sont maintenant exempts de polio. Par contre la circulation des poliovirus reste importante en Afrique de l'Ouest, en Afrique centrale et dans la corne de I'Afrique [3, 4]. Des 3 poliovirus, c'est le sérotype 1 qui reste prépondérant dans ces pays encore endémiques. Le poliovirus 2 a déjà presque disparu.

\section{Le point sur la situation française}

A la suite de la mise en place de la vaccination généralisée, rendue obligatoire en 1964, la poliomyélite a rapidement reculé en France, où elle a disparu depuis plus de 10 ans. Le dernier cas autochtone date de 1989. Le dernier cas importé, en 1995, est particulièrement instructif: il s'agissait d'un adulte de 27 ans, très partiellement vacciné (non vacciné dans I'enfance, il avait reçu une seule injection de vaccin inactivé 2 ans auparavant), qui a été hospitalisé en réanimation, à son retour d'un séjour en Côte d'Ivoire, pour une tétraplégie avec détresse respiratoire. N'ayant été évoqué que tardivement, le diagnostic de poliomyélite n'a pu être confirmé que par la sérologie (avec une séroconversion vis-à-vis du poliovirus 1). Ce cas met l'accent sur le risque $d^{\prime}$ importation, en provenance notamment des pays d'Afrique occidentale et centrale, régions d'endémie persistante, avec lesquelles la France entretient d'importants échanges. II en résulte qu'une vigilance particulière doit être maintenue vis-à-vis de ce risque d'importation des poliovirus.

La stratégie de lutte, récemment redéfinie par le Conseil Supérieur $d^{\prime}$ 'Hygiène publique, $d$ 'après le plan d'action élaboré au sein de la Commission nationale de certification de l'éradication de la poliomyélite*, repose à la fois sur le maintien à un niveau très élevé (autour de $97 \%$ ) de la couverture vaccinale des jeunes enfants, vaccinés presque exclusivement par le vaccin injectable, et sur le renforcement de la surveillance.

La surveillance clinique de la poliomyélite paralytique nécessite une vigilance accrue des cliniciens impliqués, notamment des neurologues, des pédiatres, des réanimateurs, dont l'immense majorité n'a jamais observé personnellement de cas de poliomyélite, maladie devenue rare depuis la généralisation de la vacci-

Commission composée de D. Antona M. Aymard, S. Dubrou, M. Dumas, N. Guérin, D. Lévy-Bruhl, JC Raphaël, I. Rebière, M. Rey, M. Tardieu. Secrétariat: R. Manigat (DGS). nation, au cours des années 1960. $C^{\prime}$ est pourquoi une mise au point a été diffusée en 1998 à I'intention de ces cliniciens [5]. Tout cas suspect de poliomyélite (paralysie flasque aiguë non traumatique motrice pure) doit maintenant, quel que soit l'âge du patient, être immédiatement déclaré à la DDASS et faire l'objet dune investigation épidémiologique (retour d'un pays endémique, vaccination absente ou insuffisante) et virologique. Quant à la notification et l'investigation virologique systématiques de tous les cas de paralysie flasque aiguë, telle qu'elle a été recommandée par l'OMS, elle n'a pas été mise en place en France, de même que dans plusieurs pays d'Europe occidentale, qui ont estimé avoir une infrastructure et une compétence médicales suffisantes pour détecter une suspicion de poliomyélite et distinguer celle-ci d'un syndrome de Guillain-Barré ou de cas de myélite transverse ou de compression médullaire.

Le renforcement de la surveillance virologique a été focalisé en France sur la surveillance des entérovirus, au niveau des laboratoires ayant la capacité $d$ 'isoler ces virus, et faisant partie du Réseau national de surveillance des Entérovirus, coordonné par I'Institut de Veille Sanitaire (Saint-Maurice) et par le Centre national de Référence des Entérovirus (Lyon). Ce programme concerne d'abord les cas cliniques suspects d'infection poliomyélitique: paralysies flasques aiguës, mais aussi méningites lymphocytaires. Il est basé sur la recherche d'entérovirus, par culture sur cellules appropriées, dans deux prélèvements de selles successifs (à 24-48 h d'intervalle), suivie du typage d'un éventuel poliovirus identifié. L'usage quasi exclusif du vaccin inactivé simplifie les investigations virologiques, la découverte d'un poliovirus "Sabin like» se limitant à des importations, en provenance de pays qui utilisent le vaccin oral. La recherche d'entérovirus dans le LCR est de plus en plus réalisée par PCR. Une double sérologie, à une à deux semaines d'intervalle, peut aussi être effectuée. A ce programme a été ajoutée une surveillance de la circulation des enterovirus dans l'environnement. Les excreta de 9 millions d'habitants de la région parisienne, parmi lesquels un grand nombre de voyageurs et 
d'immigrés, sont régulièrement analysés par le Laboratoire d'Hygiène de la ville de Paris. Parmi de très nombreux enterovirus détectés par ce programme quelques rares poliovirus importés sont parfois isolés. Le dernier poliovirus détecté ainsi, en dehors de tout cas clinique identifié, date de 1998. Aucun poliovirus n'a été détecté en France en 1999.

\section{Achever l'éradication et planifier la postéradication}

Pour finir de gagner la guerre mondiale contre la poliomyélite, il faut à la fois maintenir son élimination des pays déclarés exempts, et renforcer la lutte dans les pays où circulent encore les poliovirus. Les principaux réservoirs de poliovirus sont actuellement I'Inde, le Pakistan et le Bangladesh en Asie, le Nigeria, I'Éthiopie et le République démocratique du Congo en Afrique. S'y ajoutent les pays en guerre, tels I'Afghanistan, le Soudan, la Sierra Leone, I'Angola. En fait l'ensemble du sous-continent indien, de I'Afrique occidentale et centrale et de la corne de l'Afrique est encore considérée comme endémique. Dans ces pays généralement sous-équipés en infrastructures sanitaires, le programme est obéré par I'insuffisance de la détection des cas cliniques de paralysie flasque aiguë et de l'investigation virologique, malgré la mise en place d'un réseau mondial de laboratoires de référence accrédités $[3,4]$.

Espérée pour I'an 2000, I'achèvement de l'éradication mondiale de la poliomyélite va donc encore demander quelques années. Combien faudra-t-il de temps pour éliminer la polio de ses foyers asiatiques et africains résiduels? La certification de l'éradication est établie à l'échelle régionale avant d'être proclamée à l'échelle mondiale. Ce n'est qu'après trois années au moins totalement exemptes de poliomyélite que peut être envisagée la certification, sous condition d'une bonne surveillance. Dans la région européenne la certification de l'élimination de la poliomyélite pourrait être prononcée en 2003, peu après celle concernant la région du Pacifique occidental.

Parmi les problèmes posés par la dernière étape de l'effort d'éradication, celui de son financement n'est pas le moindre. Trouvera-t-on les fonds nécessaires? Les donateurs ne risquent-ils pas de se démotiver?

Autre problème: quand va-t-on s'autoriser à interrompre la vaccination par le vaccin oral? Celle-ci est remise en question par un nombre croissant de pays industrialisés (dont les États-Unis), qui n'acceptent plus le risque, même faible, d'accidents paralytiques postvaccinaux, et qui remplacent de plus en plus le vaccin oral par le vaccin inactivé. D'autre part il ne semble pas raisonnable, après avoir obtenu l'élimination des poliovirus sauvages, de continuer à déverser des poliovirus vaccinaux sur la population et dans I'environnement. Par ailleurs la suppression de la circulation des poliovirus vaccinaux simplifierait la surveillance virologique.

Enfin se pose le problème de l'avenir des trois poliovirus, comme a été posé celui du virus de la variole après l'éradication mondiale de celle-ci (problème d'ailleurs incomplètement résolu). Après la disparition des poliovirus de leur réservoir humain, il n'y aura plus qu'une source potentielle de contamination, les laboratoires détenteurs de virus. Un plan de "confinement» a donc été défini par I'OMS. II comporte trois étapes. Après l'étape, encore en cours, de pré-éradication, où la manipulation des entérovirus se fait dans des laboratoires équipés en P2, s'ouvrira l'étape de post-éradication mondiale, un an après la détection du dernier poliovirus sauvage, au cours de laquelle la manipulation des poliovirus sera restreinte aux laboratoires disposant de la sécurité P3. Par la suite, après la cessation de toute vaccination par le vaccin polio oral, l'ensemble des poliovirus devront être confinés dans les rares laboratoires disposant d'une haute sécurité de niveau P $4[6,7]$. Ce programme ambitieux rencontrera probablement quelques difficultés d'application. II faudra de toute façon maintenir, à l'échelle mondiale, une surveillance étroite des entérovirus en circulation.

\section{RÉFÉRENCES}

1. Rey M, Guérin N. Poliomyélite. Encycl Méd Chir (Elsevier, Paris), Pédiatrie 4-310A-10, Maladies Infectieuses, 8-O58-A-10, 1997; 8 p.

2. Aylward RB, Hull HF, Cochi SL, Sutter RW, Olivé JM, Melgaard B. Disease eradication as a public health strategy: a case study of poliomyelitis eradication. Bull WHO 2000; 78: 285-97.

3. OMS. Global polio eradication. Progress 1999. WHO/polio/OO.O3. Genève Organisation Mondiale de la Santé, avril 2000: 21 p.

4. OMS. Progrès vers l'éradication mondiale de la poliomyélite, 1999. Relevé Epidémiol Hebd 2000; 17 : 133-44.

5. Commission nationale de certification de l'éradication de la poliomyélite. Vers la fin de la poliomyélite. Mise en place de la dernière phase du programme d'éradication. Arch Pediatr 1998 : 5. Lettre du Neurologue 1998; 5-II : 269-70.

6. OMS. WHO global action plan for laboratory containment of wild polioviruses. Genève: Organisation Mondiale de la Santé, décembre1999; 33 p.

7. OMS. Guidelines for Implementation of laboratory containment of wild poliovirus. European Health 21 target 7. World Health

\section{TIRÉS À PART}

M. Rey. 